\title{
Kybernetika
}

\section{Hamed Tirandaz}

Chaos synchronization of TSUCS unified chaotic system, a modified function projective control method

Kybernetika, Vol. 54 (2018), No. 4, 829-843

Persistent URL: http://dml.cz/dmlcz/147427

\section{Terms of use:}

(C) Institute of Information Theory and Automation AS CR, 2018

Institute of Mathematics of the Czech Academy of Sciences provides access to digitized documents strictly for personal use. Each copy of any part of this document must contain these Terms of use.

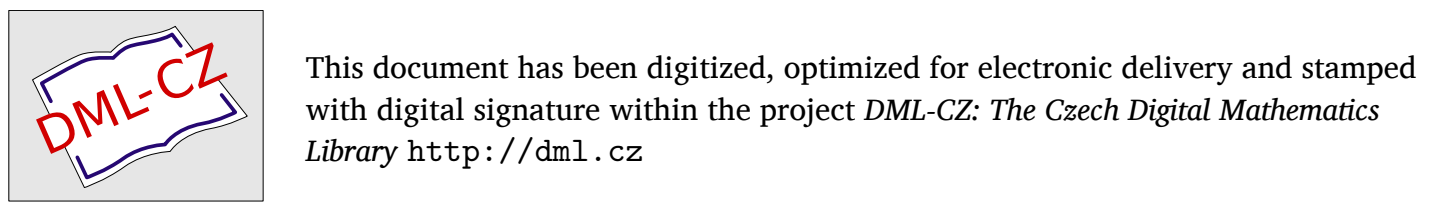




\title{
CHAOS SYNCHRONIZATION OF TSUCS UNIFIED CHAOTIC SYSTEM, A MODIFIED FUNCTION PROJECTIVE CONTROL METHOD
}

\author{
HAMED TIRANDAZ
}

The synchronization problem of the three-scroll unified chaotic system (TSUCS) is studied in this paper. A modified function projective synchronization (MFPS) method is developed to achieve this goal. Furthermore, the only parameter of the TSUCS unified chaotic system is considered unknown and estimated with an appropriate parameter estimation law. MFPS method is investigated for both identical and non-identical chaotic systems. Lyapunov stability theorem is utilized to verify the proposed feedback control laws and validate the proposed synchronization scheme. Finally, some numerical simulations are presented to assess the effectiveness of the theoretical discussions.

Keywords: chaos synchronization, three-scroll unified chaotic system (TSUCS), modified function projective synchronization (MFPS), nonlinear dynamics

Classification: 93Cxx, 93Dxx, 65Pxx

\section{INTRODUCTION}

Chaotic phenomenon has been observed and studied in various fields of engineering and science such as physics, mechanics, ecology, biology, economy, secure communication and so on [3, 14, 31, 36, 43.

The challenge of synchronization between two chaotic systems has attracted a lot of attention by many researchers, in recent years. Chaos is an unpredictable, boundedness phenomenon that is much sensitive to the initial values of the dynamical state variables. Chaos generally appears in nonlinear dynamical systems. Since Lorenz [15] in 1963 has discovered his 3D chaotic attractor, many chaotic systems have been found and studied by the researchers. Chen's system [4, Lu's system [16], Chua's system [5] are some prevalent investigated systems. Furthermore, some unified chaotic systems that benefit to the advantages of some chaotic systems have been extended. For example, L in [17, uncertain fractional-order unified chaotic system (UFOUCS) in [22, Elhadj in 8], TSUCS in [23] are some of the proposed unified chaotic systems. The readers can refer to reference [28, 29] for more information about chaotic system and how create.

The objective of chaos synchronization is to track the motion trajectories of a drive chaotic system state variables by the motion trajectories of a response chaotic system.

DOI: 10.14736/kyb-2018-4-0829 
The pioneering investigation on chaotic systems and their synchronization was carried out in 1990 by Ott et al in 21] and by Pecora in [25]. After that, several control schemes have been developed to synchronize the behavior of the chaotic systems. Active method [20, 26, adaptive method [1, 9, 18, 42, impulsive method [13, projective method 6. 32, lag method [27, sliding mode method [2, 30, linear feedback control 33. and backstepping control method [41] are some of the prevalent synchronization methods.

Among the several developed approaches for synchronization of chaotic systems, synchronization related to the projective methods have considerably attention because the drive-response synchronization can be aligned up to a scaling factor . Until now, various projective schemes has proposed to solve the synchronization between two chaotic systems either identical or non-identical ones. In [19, a projective synchronization (PS) is introduced for synchronization of the two identical Lorenz system. Then in [12], controlling of the chaotic systems performed via developing a modified projective synchronization (MPS) method up to a constant scaling factor . After that, another projective method, function projective synchronization (FPS) method was extended to synchronize drive-response systems up to a matrix scaling factor, instead of constant factor. Recently, a new projective synchronization method, modified function projective synchronization (MFPS) was introduced by Du et al in [7] to synchronize drive-response systems up to a matrix scaling function $\lambda(t)=\operatorname{diag}\left(\lambda_{1}(t), \lambda_{2}(t), \cdots, \lambda_{n}(t)\right)$. It can be clearly shown that the complete synchronization, anti-synchronization, PS and MPS are special cases of the MFPS method.On the other hand, there exist minor schemes devoted to synchronization of a unified chaotic system either identical or non-identical systems (ones). The synchronization problem between a typical chaotic system and a unified system is the subject of some papers as 35 , 37. In addition, synchronization of the unified systems has studied by some researchers. Active pinning method in [22, adaptive control method in [40, sliding mode controller in [24] and [10, mixed sliding $/ H_{2} / H_{\infty}$ method in 39, Impulsive control method in [11] and Q-S control method [38] are some of the investigated methods for synchronization of the unified chaotic systems. However, there is not any published article concentrating on MFPS of unified chaotic systems, which is the subject of this paper.

The majority of the investigated schemes for synchronization of chaotic systems focus on systems with parameters that are considered definite beforehand. However, in real world problems, the parameters of the chaotic systems are influenced by some external forces that are unknown or uncertain. So the focus of this paper is on adaptive synchronization methods to address this problem.

Motivated by the above discussions, this article drives some results on adaptive MFPS of two identical/non-identical TSUCS unified chaotic systems with unknown parameters. Some highlights of this paper are as follows:

- Chaotic behavior of the TSUCS unified chaotic system is studied.

- Appropriate adaptive - MFPS feedback controllers are designed for the identical/ non-identical TSUCS unified synchronization.

- Lyapunov stability function is used to realize synchronization approaches.

- Computer simulations verify the effectiveness of the synchronization approaches. 
The reminder of this paper is organized as follows: In Section 2, a MFPS controller is designed to synchronize the identical TSUCS unified chaotic system by a MFPS method. Then, in Section 3, non-identical synchronization of the TSUCS unified chaotic system and Lü unified chaotic system is addressed. In each section, some numerical simulations are performed to assess the proposed controlling method. Finally, some concluding remarks are given in Section 4 .

\section{IDENTICAL SYNCHRONIZATION OF THE TSUCS SYSTEM}

This section studies the synchronization problem of the identical three-scroll unified chaotic system (TSUCS) with unknown system parameter. A new MFPS feedback controller is designed in order to synchronize two identical TSUCS unified chaotic systems. The theoretical discussion and numerical simulations are performed to assess the effectiveness of the proposed controller.

The TSUCS unified chaotic system recently introduced by Pan et al in [23] as a new three-scroll unified chaotic system is as follows:

$$
\begin{aligned}
& \dot{x}_{1}=20(\theta+2)\left(x_{2}-x_{1}\right)+\frac{6 \theta+4}{25} x_{1} x_{3} \\
& \dot{x}_{2}=(55-90 \theta) x_{1}+5(\theta+4) x_{2}-x_{1} x_{3} \\
& \dot{x}_{3}=\frac{-13}{20} x_{1}^{2}+x_{1} x_{2}+\left(\frac{11}{6}-\theta\right) x_{3}
\end{aligned}
$$

where $\dot{x_{1}}, \dot{x_{2}}, \dot{x_{3}}$ stands for the time derivative of the state variables of the system $x_{1}, x_{2}, x_{3}$, respectively; and $\theta$ is the only positive parameter of the TSUCS system which is bounded as $\theta \in[0,1]$.

The only parameter $\theta$ of the dynamic system (1) determine its behavior. When $\theta \in\left[0, \frac{55}{90}\right)$, the system behaves like as a Lorenz system and would have two Lyapanov exponete values: $L=\{0.24263,0.3001\}$. The chaos attractor of the system for $\theta=0$ is shown in Figure 1. Whereas $\theta=\frac{55}{90}$, represents a Lu-like system with three Lyapanov exponents as $L \equiv\{0.24263,-0.031,-1.48120\}$. the chaotic behavior for $\theta=\frac{55}{90}$ is depicted in Figure 2. Finally, When $\theta \in\left(\frac{55}{90}, 1\right]$, it likes as a Chen system with Lyapunov exponents as: $L=\{0.697,-0.039,-3.755\}$. It's chaotic behavior is provided in Figure 3 .

The TSUCS unified chaotic system (1) will asymptotically converges to its equilibrium point $(0,0,0)$, via a designed controller which established based on MFPS control method.

The three-scroll unified chaotic system (TSUCS) described in equation (1) can be re-written as a leader system as follows:

$$
\dot{X}=A_{\theta} X^{T}+x_{1} \cdot B_{\theta} X^{T}
$$

which is consisted of two parts in its right hand side: the first part is a linear term and the second one is a nonlinear term. $X=\left(x_{1}, x_{2}, x_{3}\right)$ is vector of the state variables of the leader system and $\dot{X}$ stands for the time derivative of X. The matrixes of $A_{\theta}, B_{\theta} \in R^{3 \times 3}$ are dependent to the system parameter $\theta$, and could be defined by:

$$
A_{\theta}=\left[\begin{array}{ccc}
-20(2+\theta) & 20(\theta+2) & 0 \\
55-90 \theta & 5(\theta+4) & 0 \\
0 & 0 & \frac{11-6 \theta}{6}
\end{array}\right] \quad, \quad B_{\theta}=\left[\begin{array}{ccc}
0 & 0 & \frac{6 \theta+4}{25} \\
0 & 0 & -1 \\
\frac{-13}{20} & 1 & 0
\end{array}\right] \text {. }
$$



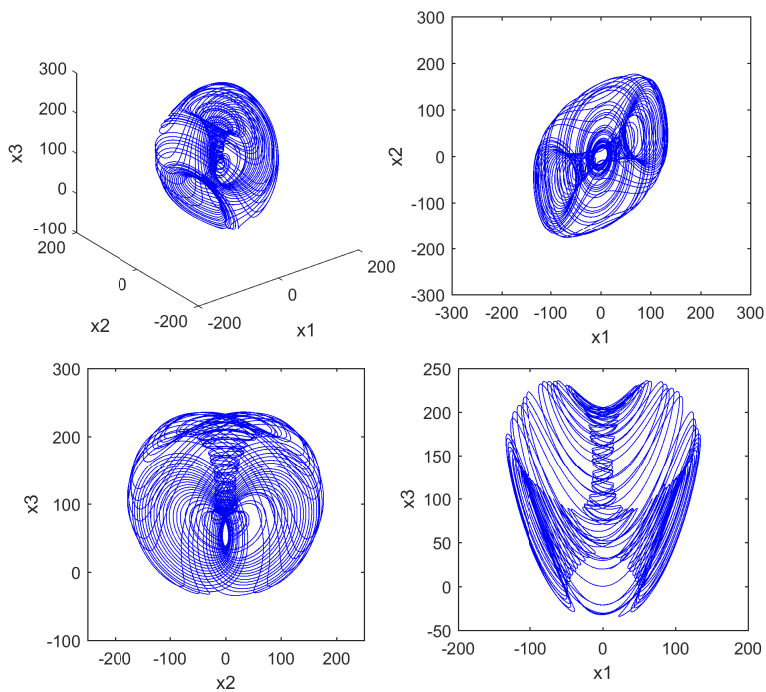

Fig. 1. Some chaotic attractors of the unified TSUCS system with $\theta=0$, lorenz-like system.
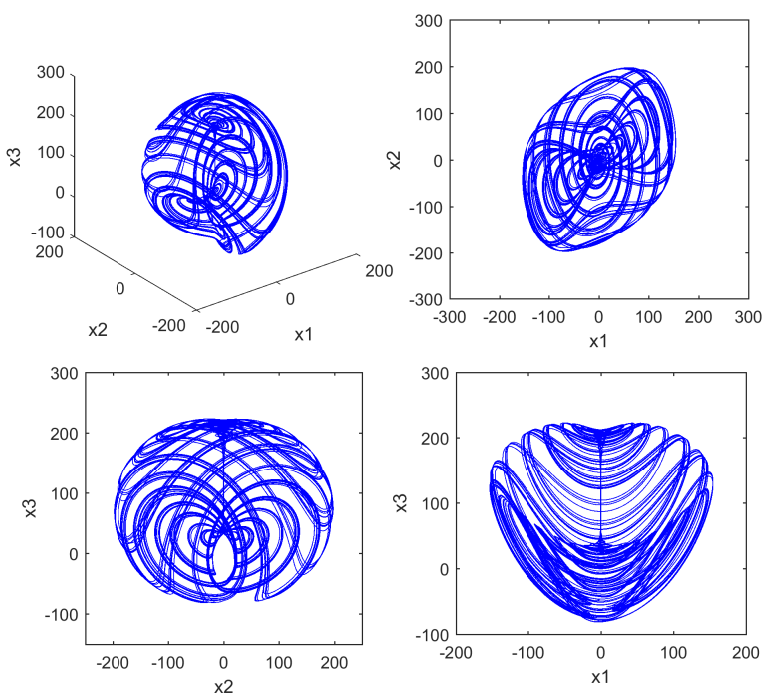

Fig. 2. Some chaotic attractors of the unified TSUCS system with $\theta=\frac{55}{90}$, Lü-like system. 

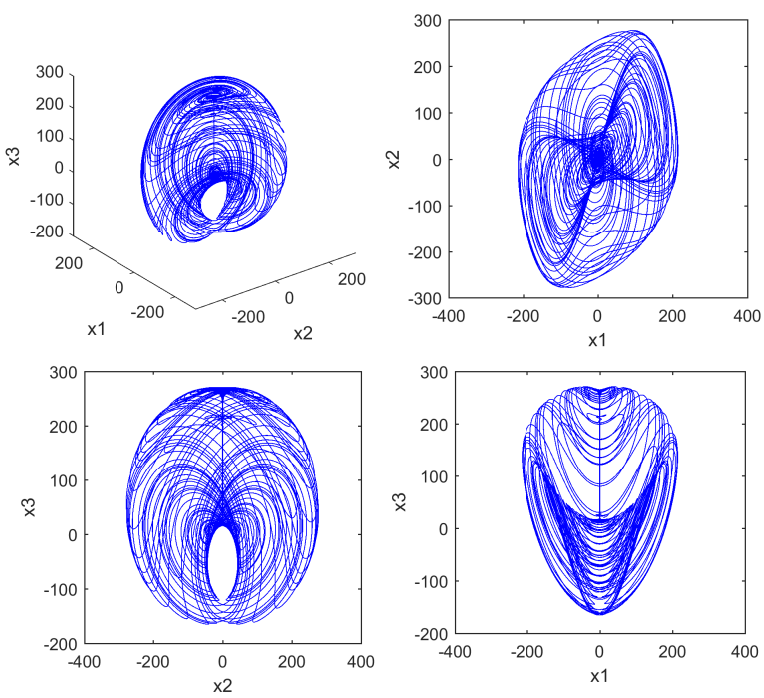

Fig. 3. Some chaotic attractors of the unified TSUCS system with $\theta=1$, Chen-like system.

These matrixes can break up into two sub-matrixes as follows:

$$
A_{\theta}=\theta A_{1}+A_{2} \quad, \quad B_{\theta}=\theta B_{1}+B_{2}
$$

where,

$$
\begin{aligned}
A_{1}=\left[\begin{array}{ccc}
-20 & 20 & 0 \\
-90 & 5 & 0 \\
0 & 0 & -1
\end{array}\right] & , A_{2}=\left[\begin{array}{ccc}
-40 & 40 & 0 \\
55 & 20 & 0 \\
0 & 0 & \frac{11}{6}
\end{array}\right] \\
B_{1}=\left[\begin{array}{lll}
0 & 0 & \frac{6}{25} \\
0 & 0 & 0 \\
0 & 0 & 0
\end{array}\right] & , B_{2}=\left[\begin{array}{ccc}
0 & 0 & \frac{4}{25} \\
0 & 0 & -1 \\
\frac{-13}{20} & 1 & 0
\end{array}\right]
\end{aligned}
$$

Then, the equations that represent the leader system can be rewritten as:

$$
\dot{X}^{T}=\left(\theta A_{1}+A_{2}\right) X^{T}+x_{1} \cdot\left(\theta B_{1}+B_{2}\right) X^{T} .
$$

Similarly, the follower system can be represented as follows, which is considered identical to the leader system $(6)$ :

$$
\dot{Y}^{T}=\left(\hat{\theta} A_{1}+A_{2}\right) Y^{T}+y_{1} \cdot\left(\hat{\theta} B_{1}+B_{2}\right) Y^{T}+U
$$

where $Y=\left(y_{1}, y_{2}, y_{3}\right)$ is the state variables vector of the follower system. The matrixes $A_{1}, A_{2}, B_{1}, B_{2} \in R^{3 \times 3}$ specify by equation $[3$. The truley parameter $\theta$ in the leader 
system 6 is replaced with the estimated parameter $\tilde{\theta}$ in the follower system (7). The symbol U implicates the control law of the closed-loop control system, which have to be designed in such way that alignment the behavior of the follower system to track the trajectory of the leader attractor.

The follower system in $(7)$ is consisted of three parts in its right-hand side, the two first parts, similar to the leader system (6), are respected to the linear and nonlinear parts, respectively and the third one $\mathrm{U}$, is equivalent to the nonlinear control law, specified in such way that two identical systems (6) and (7) can by synchronized.

The synchronization errors obtained by MFPS method between two identical leaderfollower systems (6) and (7) can be defined as:

$$
E_{p}=Y-\delta(t) X, \quad E_{\theta}=\theta-\hat{\theta}
$$

where $\delta(t)=\operatorname{diag}\left(\delta_{1}(t), \delta_{2}(t), \delta_{3}(t)\right)$ is a projective vector function; Then, the error dynamic system between leader-follower systems (6) and (7) can be achieved by time derivatives of (8) as follows:

$$
\dot{E}_{p}=\dot{Y}-\delta(t) \dot{X}-\dot{\delta}(t) X, \quad \dot{E}_{\theta}=-\dot{\hat{\theta}}
$$

Without considering any suitable feedback controller, the state variables trajectories of the follower and leader chaotic systems with different initial state values will quickly depart from each other. The objective of chaos synchronization is to design such feedback controller that can prevent such bifurcation problem. Now, the control vector and the parameter estimation can be defined based on the following illustrative theorem.

Theorem 2.1. The trajectories of the unified chaotic TSUCS system (6) with unknown parameter $(\theta)$ will track asymptotically by an identical TSUCS system, and unknown parameter $(\theta)$ will approximate by an estimated parameter $(\hat{\theta})$; for any initial state values and considering the feedback control and dynamical estimation parameter as follow:

$$
\begin{gathered}
U=-\left(\hat{\theta} A_{1}+A_{2}\right) Y^{T}-y_{1} \cdot\left(\hat{\theta} B_{1}+B_{2}\right) Y^{T}+\delta(t) A_{2} X^{T}+\delta(t) x_{1} B_{2} X^{T} \\
+\delta(t) \hat{\theta} A_{1} X^{T}+\delta(t) x_{1} \hat{\theta} B_{1} X^{T}+\delta^{\prime}(t) X-k_{1} \delta^{2}(t) E_{p} \\
\dot{\hat{\theta}}=-\delta(t) E_{p} A_{1} X^{T}-\delta(t) E_{p} x_{1} B_{1} X^{T}+k_{2}(\theta-\hat{\theta})
\end{gathered}
$$

where $k_{1}$ and $k_{2}$ are two arbitrary positive constants.

Pr o of. Consider the Lyapanov candicate function as follows:

$$
V=\frac{1}{2}\left(E_{p}^{2}+E_{\theta}^{2}\right)
$$

which is a positive definite function organized based on $E_{p}$, the system state variables 
error; and $E_{\theta}$, the parameter estimation error provided in (8). Then, we have:

$$
\begin{aligned}
\dot{V}= & E_{p} \dot{E}_{p}+E_{\theta} \dot{E}_{\theta} \\
= & E_{p}\left[A_{\hat{\theta}} Y^{T}+y_{1} B_{\hat{\theta}} Y^{T}+U-\delta(t)\left(A_{\theta} X^{T}+x_{1} B_{\theta} X\right)-\dot{\delta}(t) X\right]-(\theta-\hat{\theta}) \dot{\hat{\theta}} \\
= & E_{p}\left[\left(\hat{\theta} A_{1}+A_{2}\right) Y^{T}+y_{1}\left(\hat{\theta} B_{1}+B_{2}\right) Y^{T}+U\right. \\
& \left.\quad-\delta(t)\left(\left(A_{2}+\theta A_{1}\right) X^{T}+x_{1}\left(B_{2}+\theta B_{1}\right) X^{T}\right)-\dot{\delta}(t) X\right]-(\theta-\hat{\theta}) \dot{\hat{\theta}} \\
= & -\delta(t) E_{p}\left[(\theta-\hat{\theta}) A_{1} X^{T}+x_{1}(\theta-\hat{\theta}) B_{1} X^{T}+k_{1} \delta(t) E_{p}\right]-(\theta-\hat{\theta}) \dot{\hat{\theta}} \\
= & -k_{1} \delta^{2}(t) E_{p}^{2}+(\theta-\hat{\theta})\left[-\delta(t) E_{p} A_{1} X^{T}-\delta(t) E_{p} x_{1} B_{1} X^{T}-\dot{\hat{\theta}}\right] \\
= & -k_{1} \delta^{2}(t) E_{p}^{2}-k_{2}(\theta-\hat{\theta})^{2}<0 .
\end{aligned}
$$

Hence, the constructed Lyapanov function is always positive definite and also its derivative $\dot{V}(t)$ is always negative definite at its time domain. So the theorem is proved. Therefore the identical adaptive-MFPS method of the TSUCS unified chaotic system with unknown parameter can be achieved by dynamic equations (1), 10 and (11).

\subsection{Simulation results}

In this section, some simulations are carried out to show the synchronization between the master and slave systems. This simulations can simplify analysis of the synchronization approach. The implemented program uses the Runge-Kutta integration algorithm with considering the time step of $10^{-6}$ to solve the synchronization problem. The values of the drive state variables are initially taken as: $\left(x_{1}(0), x_{2}(0), x_{3}(0)\right)=(5,8,4)$. And also the initial values of the responsive state variables are initially selected as: $\left(y_{1}(0), y_{2}(0), y_{3}(0)\right)=(24,44,-18)$. The estimated parameter is initially taken as: $\theta=0.5$ and the positive gain constants are set as $k_{1}=2$ and $k_{2}=2$.

Figure 4 shows the trajectories of the whole state variables obtained from synchronization procedure between leader and follower TSUCS unified chaotic system with aforementioned initial conditions and modified function as $\delta(t)=\operatorname{diag}(1,1,1)$ - the complete synchronization. It can be seen from this figure that the synchronization errors between two chaotic system states converge asymptotically to zero. It means that the responsive system tracks the trajectories of the drive system nearly perfect. In addition, the estimated parameter is depicted in Figure 5. This figure demonstrates the error dwindling between the estimated parameters and their corresponding true values. Therefore, as we expect, the performance of the proposed synchronization method increases as long as the time tends to infinity.

The motion trajectories of the drive and response system are illustrated for modified function, $\delta(t)=\operatorname{diag}(0.01 t, 0.1 t, 0.2 t)$ and $\delta(t)=\operatorname{diag}(1+0.2 \sin (2 \pi t / 20), 1+$ $0.3 \sin (2 \pi t / 20), 1+0.25 \sin (2 \pi t / 20))$ in Figures 6 and 7 , respectively.

\section{NON-IDENTICAL TSUCS SYNCHRONIZATION PROBLEM}

This section is devoted to synchronization between two non-identical unified chaotic systems: the unified TSUCS chaotic system and well-known Lü unified chaotic system [16]. 

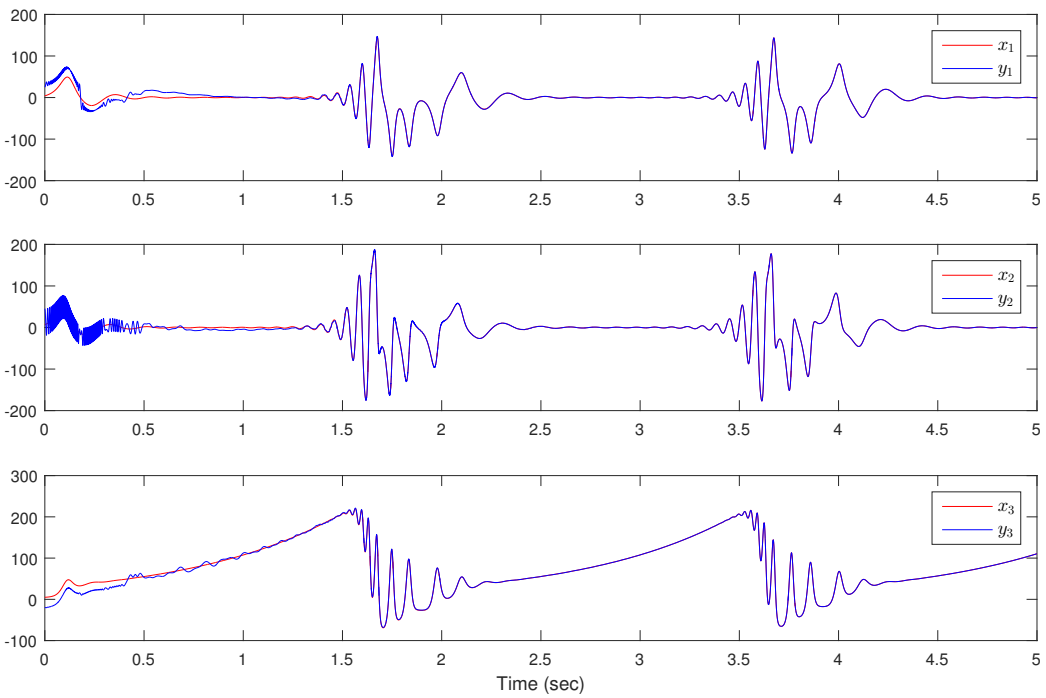

Fig. 4. Motion trajectories of the TSUCS drive-response state variables along the time with modified function $\delta(t)=\operatorname{diag}(1,1,1)-$ the complete synchronization.

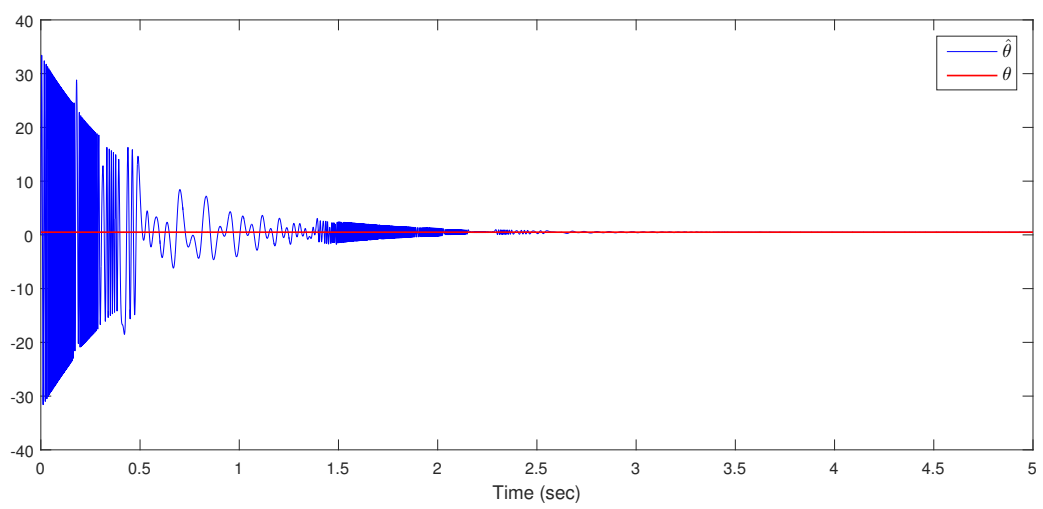

Fig. 5. TSUCS system parameter estimation $(\theta)$. 

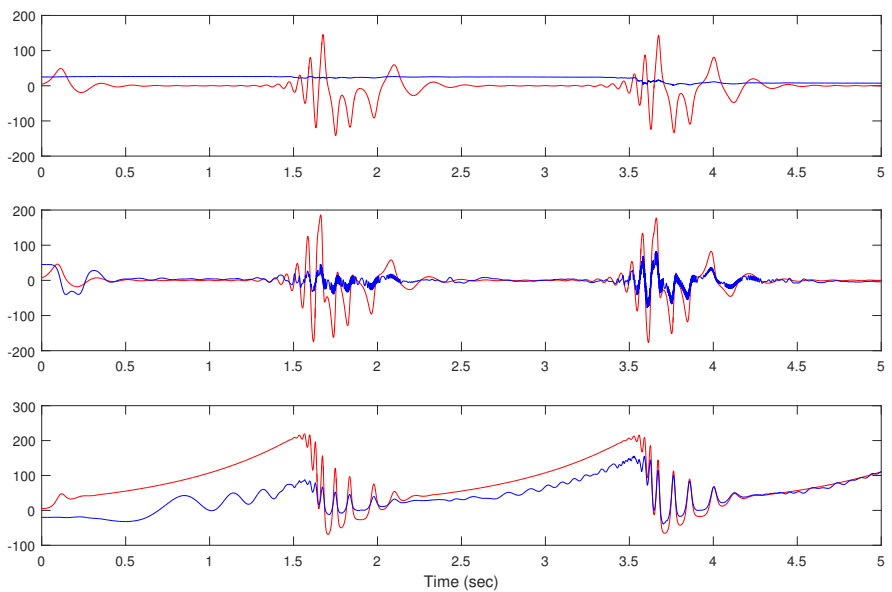

Fig. 6. Motion trajectories of the TSUCS drive-response state variables along the time with modified function

$$
\delta(t)=\operatorname{diag}(0.01 t, 0.1 t, 0.2 t) \text {. }
$$
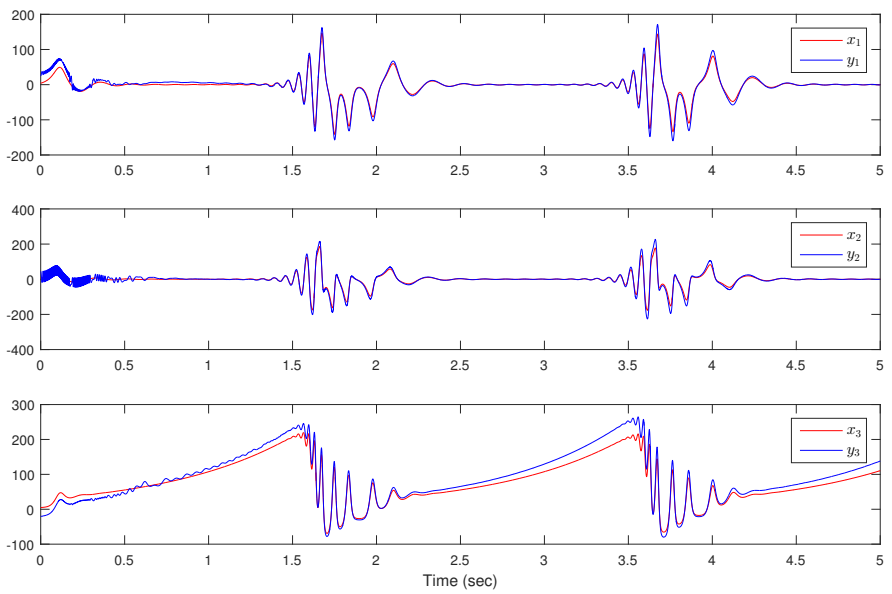

Fig. 7. Motion trajectories of the TSUCS drive-response state variables along the time with modified function $\delta(t)=\operatorname{diag}(1+0.2 \sin (2 \pi t / 20), 1+0.3 \sin (2 \pi t / 20), 1+0.25 \sin (2 \pi t / 20))$. 
Consider the unified TSUCS chaotic system (1) as leader system. Then the follower system is considered as the Lü unified chaotic system as follows:

$$
\begin{aligned}
& \dot{y_{1}}=(25 \alpha+10)\left(y_{2}-y_{1}\right)+u_{1} \\
& \dot{y_{2}}=(28-35 \alpha) y_{1}-y_{1} y_{3}+(29 \alpha-1) y_{2}+u_{2} \\
& \dot{y_{3}}=y_{1} y_{2}-\frac{\alpha+8}{3} y_{3}+u_{3}
\end{aligned}
$$

where $\alpha$ is the only parameter of the system $(\alpha \in[0,1])$. When $\alpha=0$, the chaotic system Lü is the Lorenz system. When $\alpha=0.8$, it represents the Chen system. While $\alpha=1$ shows a Lü chaotic system. Similar to the matrix representation of TSUCS system in (6), this dynamical system can be represented in a matrix form as follows:

$$
\dot{Y}^{T}=\left(\hat{\theta} C_{1}+C_{2}\right) Y^{T}+y_{1} \cdot D Y^{T}+U
$$

where $Y=\left(y_{1}, y_{2}, y_{3}\right)$ describes the state variables of the Lü unified chaotic system (14). $\hat{\theta}$ is the estimation of $\theta$, the TSUCS unified system parameter. $U \in R^{3}$ is the control feedback input, and the $C_{1}, C_{2}, D \in R^{3 \times 3}$ are the constant coefficient matrix represented as follows:

$$
C_{1}=\left[\begin{array}{ccc}
-25 & 25 & 0 \\
-35 & 29 & 0 \\
0 & 0 & -1 / 3
\end{array}\right], C_{2}=\left[\begin{array}{ccc}
-10 & 10 & 0 \\
28 & -1 & 0 \\
0 & 0 & -8 / 3
\end{array}\right], \quad D=\left[\begin{array}{ccc}
0 & 0 & 0 \\
0 & 0 & -1 \\
0 & 1 & 0
\end{array}\right]
$$

Construct the synchronization error of state variables and parameter error between the TSUCS, the leader chaotic system the Lü, the follower chaotic system by adaptiveMFPS approach represented and its dynamic error as follows

$$
E_{p}=Y-\delta(t) X, \quad E_{\theta}=\theta-\hat{\theta}
$$

where $\delta(t)=\left(\delta_{1}(t), \delta_{2}(t), \delta_{3}(t)\right)$ is a modified function vector and $\alpha$ is the parameter of the follower Lü unified system; Then, the error dynamic system between leader-follower systems (2) and (4) can be achieved by time derivatives of (5) as follows:

$$
\dot{E}_{p}=\dot{Y}-\delta(t) \dot{X}-\dot{\delta}(t) X, \quad \dot{E}_{\theta}=-\dot{\hat{\theta}} .
$$

Now the appropriate feedback control and estimated parameter $\theta$ is defined at the following theorem.

Theorem 3.1. For the given leader unified TSUCS system (6), the follower unified Lü system (15), the state variable error and parameter error (17) and their corresponding dynamical equations (18), and for any initial values, the motion trajectories of the follower system tracks the trajectories of the leader system if the feedback control input and estimated update law of the follower system parameter $\alpha$ satisfy:

$$
\begin{aligned}
U= & -\left(\hat{\theta} C_{1}+C_{2}\right) Y^{T}-y_{1} \cdot D Y^{T}+\delta(t)\left(\left(\hat{\theta} A_{1}+A_{2}\right) X^{T}+x_{1} \cdot\left(\hat{\theta} B_{1}+B_{2}\right) X^{T}\right) \\
& +\dot{\delta}(t) X^{T}-k_{1} \delta^{2}(t) E_{p}
\end{aligned}
$$


and,

$$
\dot{\hat{\theta}}=-\delta(t) E_{p}\left(A_{1} X^{T}+x_{1} B_{1} X^{T}\right)+k_{2}(\theta-\hat{\theta})
$$

where $k_{1}$ and $k_{2}$ are two arbitrary positive constants.

Proof. Construct the Lyapanov statility function as follows:

$$
V=\frac{1}{2}\left(E_{p}^{2}+E_{\theta}^{2}\right)
$$

which is positive definite. Time derivative of the $\mathrm{V}(\mathrm{t})$ along the solution of synchronization errors (17) and utilizing the control law (19) and the estimated parameter $\theta$ in (20) will be

$$
\begin{aligned}
\dot{V}= & E_{p} \dot{E}_{p}+E_{\theta} \dot{E}_{\theta} \\
= & E_{p}\left[\left(\hat{\theta} C_{1}+C_{2}\right) Y^{T}+y_{1} \cdot D Y^{T}+U\right. \\
& \left.\quad \quad \delta(t)\left(\left(\theta A_{1}+A_{2}\right) X^{T}+x_{1} \cdot\left(\theta B_{1}+B_{2}\right) X^{T}\right)-\dot{\delta}(t) X^{T}\right]-(\theta-\hat{\theta}) \dot{\hat{\theta}} \\
& \quad-\delta(t) E_{p}\left[(\theta-\hat{\theta}) A_{1} X^{T}+x_{1} \cdot(\theta-\hat{\theta}) B_{1} X^{T}+k_{1} \delta(t) E_{p}\right]-(\theta-\hat{\theta}) \dot{\hat{\theta}} \\
= & -k_{1}-\delta(t)^{2} E_{p}^{2}-k_{2}(\theta-\hat{\theta})^{2}<0 .
\end{aligned}
$$

Hence, the theorem is proved. Then, the anticipated MFPS problem is achieved.

\subsection{Numerical simulation}

This section is devoted to synchronization problem between TSUCS and Lu, two unified chaotic system via MFPS control method. The initial values of the drive and response chaotic systems are taken as: $X(0)=\left(x_{1}(0), x_{2}(0), x_{3}(0)\right)=(3,5,2)$ and $Y(0)=\left(y_{1}(0), y_{2}(0), y_{3}(0)\right)=(17,-5,15)$, respectively. The initial estimation of the drive system parameter are set as: $\theta=0.7$.

The time series behavior of the drive and response system is depicted in Figure 8 with modified function $\delta(t)=\operatorname{diag}(1+0.07 \sin (2 \pi t / 20), 1+0.2 \sin (2 \pi t / 20), 1+0.05 \sin (2 \pi t / 20)$. Furthermore, time series of the drive and response parameter estimation errors is shown in Figure 9

\section{CONCLUSION}

In this paper, the identical and non-identical synchronization problem of TSUCS unified chaotic system is studied. A MFPS controller is designed to synchronize drive and response systems. The validity of the proposed MFPS synchronization methods are verified via Lyapunov stability theorem. Finally some numerical simulations are given. The numerical simulation results show that the proposed controllers can obtain the synchronization problem in a short time, with excellent stability conditions. 

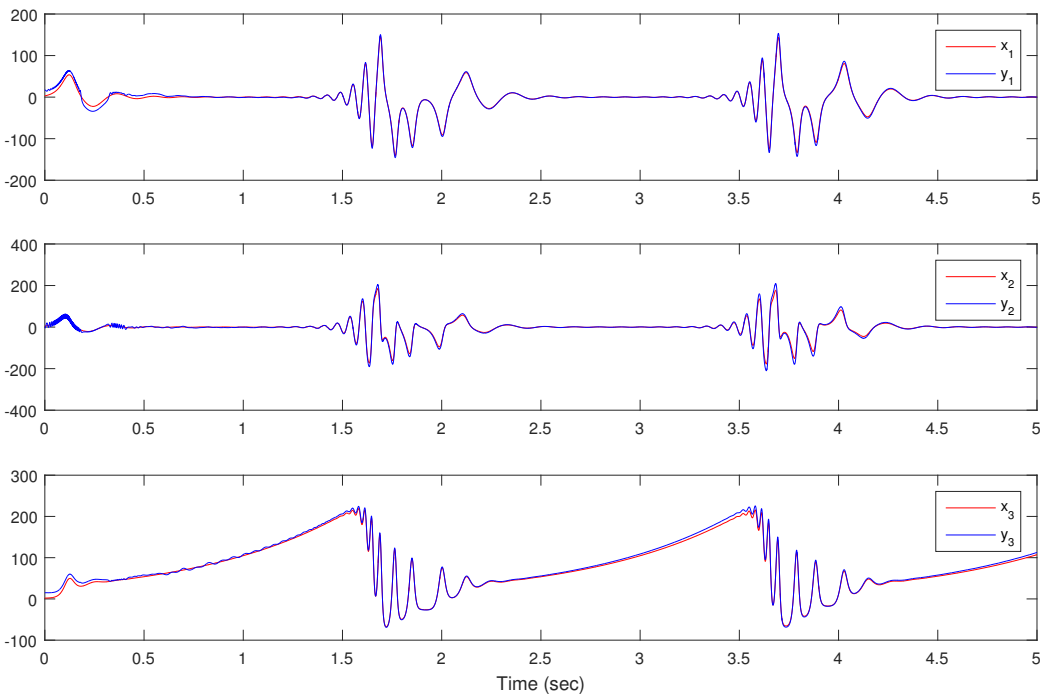

Fig. 8. Motion trajectories TSUCS and Lü drive-response state variables along the time with modified function $\delta(t)=$ $\operatorname{diag}(1+0.02 \sin (2 \pi t / 20), 1+0.2 \sin (2 \pi t / 20), 1+0.05 \sin (2 \pi t / 20))$.

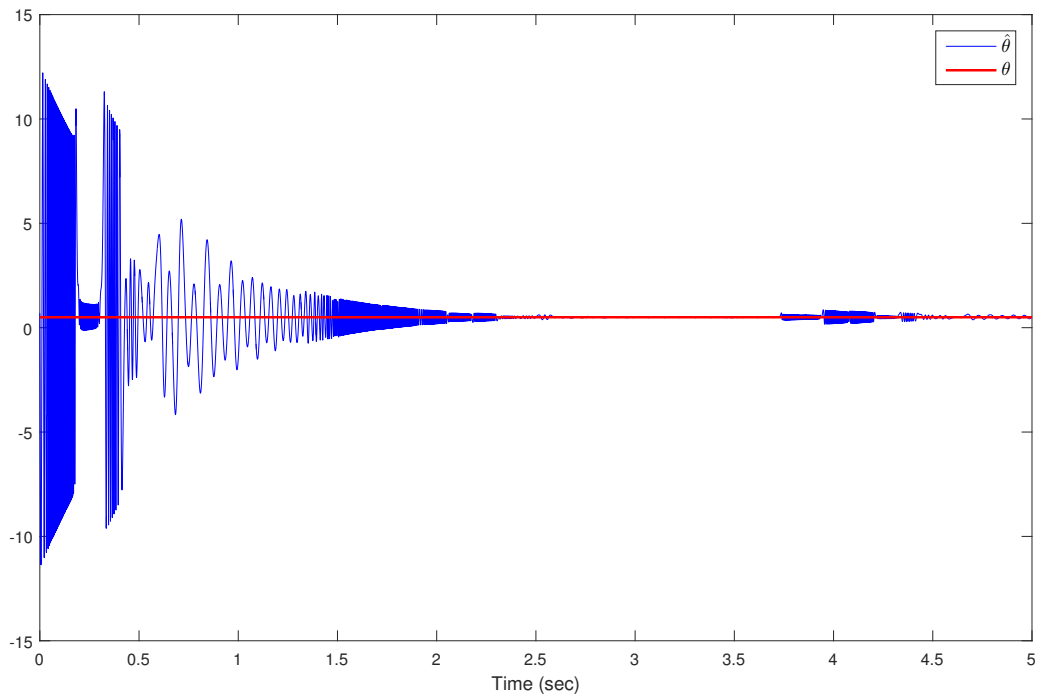

Fig. 9. Non-identical TSUCS system parameter estimation $(\theta)$. 
[1] H. Adloo and M. Roopaei: Review article on adaptive synchronization of chaotic systems with unknown parameters. Nonlinear Dynamics 65 (2011), 1-2, 141-159. DOI:10.1007/s11071-010-9880-6

[2] M. P. Aghababa and A. Heydari: Chaos synchronization between two different chaotic systems with uncertainties, external disturbances, unknown parameters and input nonlinearities. Appl. Math. Modelling 36 (2012), 4, 1639-1652. DOI:10.1016/j.apm.2011.09.023

[3] L. Cai and J. Zhou: Impulsive stabilization and synchronization of electro-mechanical gyrostat systems. Nonlinear Dynamics 70 (2012) 1, 541-549. DOI:10.1007/s11071-0120474-3

[4] G. Chen and T. Ueta: Yet another chaotic attractor. Int. J. Bifurcation Chaos 9 (1999), 07, 1465-1466. DOI:10.1142/s0218127499001024

[5] L. O. Chua and G.-N. Lin: Canonical realization of chua's circuit family. IEEE Trans. Circuits Syst. 37 (1990), 7, 885-902. DOI:10.1109/31.55064

[6] L. Chun-Lai, Z. Mei, Z. Feng, and Y. Xuan-Bing: Projective synchronization for a fractional-order chaotic system via single sinusoidal coupling. Optik - Int. J. Light Electron Optics 127 (2016), 5, 2830-2836. DOI:10.1016/j.ijleo.2015.11.197

[7] H. Du, Q. Zeng, and C. Wang: Modified function projective synchronization of chaotic system. Chaos, Solitons, Fractals 42 (2009), 4, 2399-2404. DOI:10.1016/j.chaos.2009.03.120

[8] Z. Elhadj and J.C. Sprott: The unified chaotic system describing the lorenz and chua systems. Facta Univers., Ser. Electronics and Energetics 23 (2010), 3, 345-355. DOI:10.2298/fuee1003345e

[9] K.-S. Hong et al.: Adaptive synchronization of two coupled chaotic hindmarsh-rose neurons by controlling the membrane potential of a slave neuron. Appl. Math. Modelling 37 (2013), 4, 2460-2468. DOI:10.1016/j.apm.2012.06.003

[10] Y.-Y. Hou, B.-Y. Liau, and H.-C. Chen: Synchronization of unified chaotic systems using sliding mode controller. Math. Problems Engrg. 2012.

[11] C. Hu and H. Jiang: Stabilization and synchronization of unified chaotic system via impulsive control. In: Abstract and Applied Analysis, Hindawi Publishing Corporation 2014, pp. 1-8. DOI:10.1155/2014/369842

[12] G.-H. Li: Modified projective synchronization of chaotic system. Chaos, Solitons, Fractals 32 (2007), 5, 1786-1790. DOI:10.1016/j.chaos.2005.12.009

[13] C. Li, X. Liao, and X. Zhang: Impulsive synchronization of chaotic systems. Chaos: An Interdisciplinary J. Nonlinear Sci. 15 (2005), 2, 023104. DOI:10.1063/1.1899823

[14] H. Liang, Z. Wang, Z. Yue and R. Lu: Generalized synchronization and control for incommensurate fractional unified chaotic system and applications in secure communication. Kybernetika 48 (2012), 2, 190-205.

[15] E. N. Lorenz: Deterministic nonperiodic flow. J. Atmospher. Sci. 20 (1963), 2, 130-141. DOI:10.1175/1520-0469(1963)020;0130:dnfi2.0.co;2

[16] J. Lü and G. Chen: A new chaotic attractor coined. Int. J. Bifurcation Chaos 12 (2002), 03, 659-661. DOI:10.1142/s0218127402004620

[17] J. Lü, G. Chen, D. Cheng, and S. Čelikovský: Bridge the gap between the Lorenz system and the Chen system. Int. J. Bifurcation Chaos 12 (2002), 12, 2917-2926. DOI:10.1142/s021812740200631x 
[18] T. Ma, J. Zhang, Y. Zhou, and H. Wang: Adaptive hybrid projective synchronization of two coupled fractional-order complex networks with different sizes. Neurocomputing 164 (2015), 182-189. DOI:10.1016/j.neucom.2015.02.071

[19] R. Mainieri and J. Rehacek: Projective synchronization in three-dimensional chaotic systems. Phys. Rev. Lett. 82 (1999), 15, 3042. DOI:10.1103/physrevlett.82.3042

[20] H.S. Nik, J. Saberi-Nadjafi, S. Effati, and R.A. Van Gorder: Hybrid projective synchronization and control of the baier-sahle hyperchaotic flow in arbitrary dimensions with unknown parameters. Appl. Math. Comput. 248 (2014), 55-69. DOI:10.1016/j.amc.2014.08.108

[21] E. Ott, C. Grebogi, and J. A. Yorke: Controlling chaos. Phys. Rev. Lett. 64 (1990), 11, 1196-1199. DOI:10.1103/physrevlett.64.1196

[22] L. Pan, W. Zhou, J. Fang, and D. Li: Synchronization and anti-synchronization of new uncertain fractional-order modified unified chaotic systems via novel active pinning control. Comm. Nonlinear Sci. Numer. Simul. 15 (2010), 12, 3754-3762. DOI:10.1016/j.cnsns.2010.01.025

[23] L. Pan, W. Zhou, J. Fang, and D. Li: A new three-scroll unified chaotic system coined. Int. J. Nonlinear Sci. 10 (2010), 4, 462-474.

[24] J.H. Park: Adaptive synchronization of a unified chaotic system with an uncertain parameter. Int. J. Nonlinear Sci. Numer. Simulation 6 (2005), 2, 201-206. DOI:10.1515/ijnsns.2005.6.2.201

[25] L. M. Pecora and T. L. Carroll: Synchronization in chaotic systems. Phys. Rev. Lett. 64 (1990), 8, 821. DOI:10.1103/physrevlett.64.821

[26] H. Richter: Controlling chaotic systems with multiple strange attractors. Physics Letters A 300 (2002), 2, 182-188. DOI:10.1016/s0375-9601(02)00183-4

[27] M. G. Rosenblum, A.S. Pikovsky, and J. Kurths: From phase to lag synchronization in coupled chaotic oscillators. Phys. Rev. Lett. 78 (1997), 22, 4193. DOI:10.1103/physrevlett.78.4193

[28] C. Shen, S. Yu, J. Lü, and G. Chen: A systematic methodology for constructing hyperchaotic systems with multiple positive Lyapunov exponents and circuit implementation. IEEE Tran. Circuits Systems I 61 (2014), 3, 854-864. DOI:10.1109/tcsi.2013.2283994

[29] C. Shen, S. Yu, J. Lü, and G. Chen: Designing hyperchaotic systems with any desired number of positive Lyapunov exponents via a simple model. IEEE Trans. Circuits Systems I 61 (2014), 8, 2380-2389. DOI:10.1109/tcsi.2014.2304655

[30] J. Sun, Y. Shen, X. Wang, and J. Chen: Finite-time combination-combination synchronization of four different chaotic systems with unknown parameters via sliding mode control. Nonlinear Dynamics 76 (1) (2014) 383-397. DOI:10.1007/s11071-013-1133-z

[31] S. Tan, Y. Wang, and J. Lü: Analysis and control of networked game dynamics via a microscopic deterministic approach. IEEE Trans. Automat. Control 61 (2016), 12, 41184124. DOI:10.1109/tac.2016.2545106

[32] H. Tirandaz: On adaptive modified projective synchronization of a supply chain management system. Pramana 89 (2017), 6. DOI:10.1007/s12043-017-1482-0

[33] H. Tirandaz, S. Saiedi Aminabadi, and H. Tavakoli: Chaos synchronization and parameter identification of a finance chaotic system with unknown parameters, a linear feedback controller. In: Alexandria Engineering Journal, 2017. DOI:10.1016/j.aej.2017.03.041 
[34] Q. Wang, S. Yu, C. Li, J. Lü, X. Fang, C. Guyeux, and J. M. Bahi: Theoretical Design and FPGA-based implementation of higher-dimensional digital chaotic systems. IEEE Trans. Circuits Systems I 63 (2016), 3, 401-412. DOI:10.1109/tcsi.2016.2515398

[35] X. Wu, Z.-H. Guan, and T. Li: Chaos synchronization between unified chaotic system and genesio system. In: International Symposium on Neural Networks, Springer, 2007, pp. 8-15. DOI:10.1007/978-3-540-72393-6_2

[36] X. Wu, J. Li, and R. K. Upadhyay: Chaos control and synchronization of a three species food chain model via Holling functional response. Int. J. Comput. Math. 87 (2010) 199-214. DOI:10.1080/00207160801993232

[37] H. Wu, B. L. Xu, C. Fan, and X. Y. Wu: Chaos synchronization between unified chaotic system and Rossler system. In: Applied Mechanics and Materials 321, Trans. Tech. Publ. 2013, pp. 2464-2470. DOI:10.4028/www.scientific.net/amm.321-324.2464

[38] Z. Yan: Chaos Q-S synchronization between Rossler system and the new unified chaotic system. Phys. Lett. A 334 (2005), 4, 406-412. DOI:10.1016/j.physleta.2004.11.042

[39] O. Yazdanbakhsh, S. Hosseinnia, and J. Askari: Synchronization of unified chaotic system by sliding mode/mixed h 2/h control. Nonlinear Dynamics 67 (2012), 3, 1903-1912. DOI:10.1007/s11071-011-0117-0

[40] Y. Yu: Adaptive synchronization of a unified chaotic system. Chaos, Solitons, Fractals 36 (2008), 2, 329-333. DOI:10.1016/j.chaos.2006.06.104

[41] J. Yu, B. Chen, H. Yu, and J. Gao: Adaptive fuzzy tracking control for the chaotic permanent magnet synchronous motor drive system via backstepping. Nonlinear Analysis: Real World Appl. 12 (2011), 1, 671-681. DOI:10.1016/j.nonrwa.2010.07.009

[42] X. Zhang, H. Zhu, and H. Yao: Analysis and adaptive synchronization for a new chaotic system. J. Dynamical Control Systems 18 (2012), 4, 467-477. DOI:10.1007/s10883-0129155-2

[43] Q. Zhao and H. Yin: Gbits/s physical-layer stream ciphers based on chaotic light. Optik, Elsevier 124 (2013) 15, 2161-2164. DOI:10.1016/j.ijleo.2012.06.075

Hamed Tirandaz, Electrical and Computer Engineering Faculty. Hakim Sabzevari University.

e-mail: tirandaz@hsu.ac.ir 